\title{
Proton beam velocity distributions in an interplanetary coronal mass ejection
}

\author{
E. Marsch ${ }^{1}$, S. Yao ${ }^{2}$, and C.-Y. Tu ${ }^{2}$ \\ ${ }^{1}$ Max-Planck-Institut für Sonnensystemforschung, Max-Planck-Straße 2, 37191 Katlenburg-Lindau, Germany \\ ${ }^{2}$ Department of Geophysics, Peking University, 100871 Beijing, China
}

Received: 22 October 2008 - Revised: 7 January 2009 - Accepted: 27 January 2009 - Published: 19 February 2009

\begin{abstract}
The plasma and magnetic-field instruments on the Helios 2 spacecraft, which was on 3 April 1979 located at $0.68 \mathrm{AU}$, detected an interplanetary coronal mass ejection (ICME) that revealed itself by the typical signature of magnetic field rotation. The solar wind flow speed ranged between 400 and $500 \mathrm{~km} / \mathrm{s}$. We present here some detailed proton velocity distributions measured within the ICME. These cold distributions are characterized by an isotropic core part with a low temperature, $T \leq 10^{5} \mathrm{~K}$, but sometimes reveal a broad and extended hot proton tail or beam propagating along the local magnetic field direction. These beams lasted only for about an hour and were unusual as compared with the normal ICME protons distribution which were comparatively isotropic. Furthermore, we looked into the velocity and field fluctuations in this ICME and found signatures of Alfvén waves, which might be related to the occurrence of the hot proton beams. However, it cannot be excluded that the beam originated from the Sun.
\end{abstract}

Keywords. Interplanetary physics (Solar wind plasma) Space plasma physics (Wave-particle interactions) - Solar physics, astrophysics, and astronomy (Flares and mass ejections)

\section{Introduction}

A coronal mass ejection (CME) produces the most violent type of transient solar wind and the strongest interplanetary manifestation of the active sun. A wealth of literature exists on the global plasma and field structures and the outputs of CMEs in the heliosphere at $1 \mathrm{AU}$ and beyond, e.g., in the recent book edited by Kunow et al. (2007) containing many review papers. Hitherto, Helios 1 and 2 are the only spacecraft

Correspondence to: E. Marsch (marsch@mps.mpg.de) that approached the Sun to distances of only $0.29-0.3 \mathrm{AU}$, and therefore provide a unique opportunity to study CMEs, and in particular magnetic clouds (MCs), in the inner heliosphere. For our study, we selected one MC event observed by Helios 2 at $0.675 \mathrm{AU}$ on 3 April 1979.

Quite a lot has been known for some time about the electrons in CMEs, such as the conspicuous bi-directional streaming of strahl electrons (Gosling et al., 1987; Skoug et al., 2000), which was often used to identify the closed magnetic field lines in an interplanetary CME (abbreviated as ICME). Much less has been published about the plasma microstate of the ions, other than very low ion temperatures, and the important signatures in heavy ion composition and abundance in ICMEs, for example reviewed by (WimmerSchweingruber et al., 2006).

In a short review paper about the in-situ solar wind and magnetic field signatures of ICMEs, Zurbuchen and Richardsen (2006) list, in their comprehensive Table 1, as the single kinetic feature of the thermal proton VDF the decrease of the mean temperature. In his personal account of the recent series of workshops on CMEs, Wimmer-Schweingruber (2006) summarized key open questions that are related with the solar origin of a CME and its heliospheric output in the form of an ICME. Whereas observational facts and theoretical ideas abound concerning, e.g., the heavy ion composition in ICMEs, almost nothing is known about the ion kinetic plasma state, certainly not in the same detail like this knowledge is available about the microstate of the normal solar wind (Marsch, 2006). Here we will investigate the proton velocity distribution functions (VDFs) and Alfvén waves in an ICME.

\section{Data analysis}

For our data analysis we used the ion data obtained by the Helios electrostatic plasma analyser. This instrument

Published by Copernicus Publications on behalf of the European Geosciences Union. 
(Rosenbauer et al., 1977) does not automatically discriminate protons from alpha particles. They first have to be identified and then separated from protons by techniques requiring some subtle considerations in that energy/charge domain where the spectra of both species overlap. Since the massper-charge ratio of the alpha particles is 2 , we know where to expect the alpha particles in the energy/charge spectra. We employed here the standard ion data analysis methods, as they were originally described by Marsch et al. (1982) and developed further by Heuer and Marsch (2007) and Marsch et al. (2004), in order to separate the count rates belonging to protons from those of alpha particles.

For each velocity distribution function we calculated the proton number density, $n_{p}$, flow velocity, $\mathbf{V}$, and the parallel and perpendicular temperatures based on the moment integration of the measured VDF. The measurement time of the protons typically was $10 \mathrm{~s}$, with a cadence of $40.5 \mathrm{~s}$, yielding about 90 VDFs per hour in case of continuous measurements. The proton gyroperiod in the inner heliosphere is usually shorter than a second, and thus the measured proton VDF is, by gyro-phase averaging, fairly gyrotropic, whereby its symmetry axis coincides with the independently measured, instant magnetic field direction. The components and variances of the magnetic field vector, $\mathbf{B}$, were routinely measured by the Helios magnetometer and provided by Neubauer et al. (1977), who described the analysis procedure of the magnetic field data.

\section{Observational results}

An interplanetary coronal mass ejection (ICME) was measured in situ by the plasma and magnetic-field instruments on the Helios 2 spacecraft, which was located at $0.68 \mathrm{AU}$. The associated ICME revealed the expected typical signatures, such as a magnetic field rotation indicating the magnetic flux rope of a MC. The solar wind flow speed ranged between 400 and $500 \mathrm{~km} / \mathrm{s}$ during this period. We used three distinct characteristics of a MC as usual criteria to identify its extent and temporal evolution: The magnitude of the magnetic field, $B$, that becomes stronger in the magnetic cloud, the elevation angle that changes gradually a lot during a long period of about a day, and the proton temperature which in the MC is lower than in the ambient solar wind.

Figure 1 gives a survey of the magnetic structure of the ICME and of the main solar wind parameters. From the top to the bottom panels the magnitude $B$, the azimuthal angle $\phi$ and elevation angle $\theta$ of the field are shown, together with the proton speed, density and temperature. The ICME clearly manifests itself by a rotation of the magnetic field vector, which signifies the flux-rope geometry of a MC. The classical plasma signature of ICMEs, namely a low temperature, is also obvious. We marked the ICME onset time (02:00) in Fig. 1 by the red dotted line. Across this line, there occurred sharp jumps of $B, \phi$ (azimuth angle) and $\theta$ (eleva- tion angle) when the $\mathrm{MC}$ was first encountered. The jumps are clearly visible in the respective panels. $B$ was sharply enhanced from about $10 \mathrm{nT}$ to $50 \mathrm{nT}$ near 02:00, and also the angle $\theta$ then decreased rapidly from near $90^{\circ}$ to $-90^{\circ}$. The azimuth angle, for which $\phi=0$ means that magnetic field is towards the Sun, experienced a sharp jump as well, from $-100^{\circ}$ to $100^{\circ}$ at $02: 00$. After the jump $\phi$ begins to decrease to $-100^{\circ}$ by $04: 00$. In the cloud, $B$ stayed at a high level of about $50 \mathrm{nT}$, whereas $\theta$ gradually changed from about $-90^{\circ}$ degrees to about $80^{\circ}$ degrees (19:00), and at 04:00 $\phi$ had nearly finished a circle around, but kept somewhat more stable later on.

The three-dimensional proton VDFs in the MC are for the convenience of better visibility presented in Fig. 2 only as two-dimensional contours in a plane which is determined by the magnetic field direction (thick oblique line) and the direction (positive $\mathrm{x}$-axis) radially outward from the Sun. The $\mathrm{z}$-axis, which is not shown here, results from the cross product of the unit vectors in the radial and field directions. Finally, the y-axis is defined by the vector cross product between the unit vectors in the $\mathrm{x}$ - and $\mathrm{z}$-directions. Two sequences of proton VDFs as measured, respectively, within about an hour in the ICME are shown in the top and bottom panels of Fig. 2. In association with the two periods marked by the left and right red bar in Fig. 1, we processed data from hour 6 to 7 (with 24 data points) and from 8 to 9 (with 43 data points). Because of data gaps and errors, we obtained less than the maximum possible $89 \mathrm{VDFs}$ per hour. The top panel of Fig. 2 displays gyrotropic VDFs with beam or skewness. These VDFs typically have a comparatively cold and isotropic core, but in addition a broad and elongated heat flux tail, or intermittently a resolved proton beam along the field direction which represents the symmetry axis. These beams in the top panel are in striking contrast to the isotropic distributions of the bottom panel, which appear to be the rule for the proton VDFs measured in this ICME. Concerning the separation of the alpha particles, this procedure was illustrated in Fig. 2 (1) of Marsch et al. (1982), which shows that the alpha particles are located on the x-axis far away from the protons. We used the same separation technique described there in our present paper.

The core parts of the beam VDFs have a temperature of less than $10^{5} \mathrm{~K}$. Note the higher temperature of the beam than in the core, a thermal feature that is clearly visible by much broader isodensity contours of the beam, which has a small fractional proton number density. The beam distributions appear dynamic and variable, rather than stable in shape during this time period. There are no obvious beam distributions before (from hour 17 in DOY 92 to hour 2 in DOY 93) and after the MC (from hour 9 to hour 17 in DOY 94), or in the rest of the ICME either. The differential speed between beam (or tail center) and core is about $150-200 \mathrm{~km} / \mathrm{s}$. This is a sizable fraction of the local Alfvén speed, which has an average value of $280 \mathrm{~km} / \mathrm{s}$ for the top four beam proton VDFs. 


\section{Helios-2}

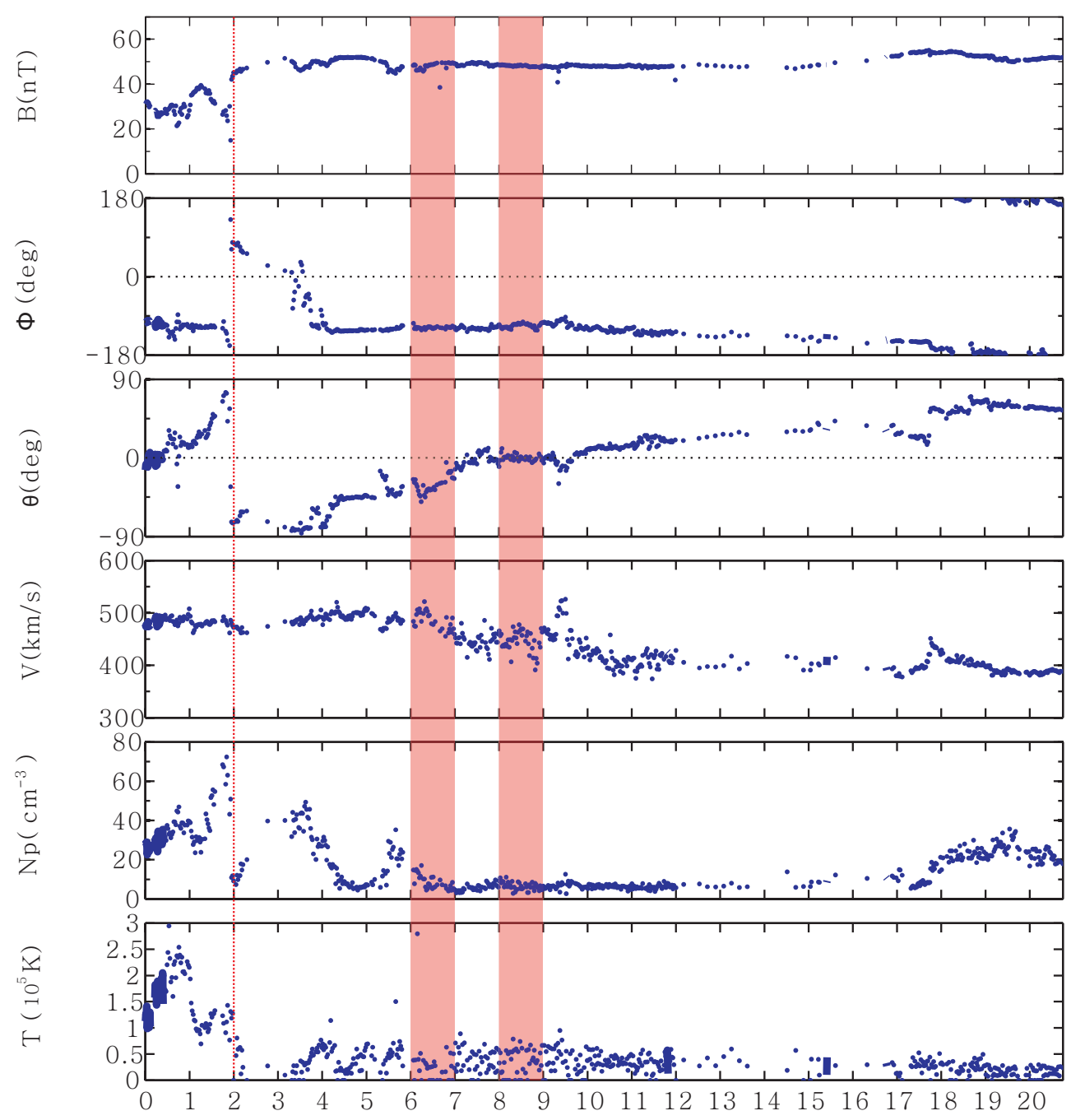

Hours after 00:00 UT,3 April (DOY 93) 1979

Fig. 1. Magnetic field associated with an interplanetary coronal mass ejection (ICME) as observed by Helios 2 on 3 April 1979 at 0.675 AU. From top to bottom the magnitude, azimuthal angle and elevation angle of the magnetic field are shown. The following three panels give the plasma parameters solar wind speed (protons), proton density and temperature. The ICME manifests itself by the classical rotation (start is indicated by the red vertical dotted line) of the field, which is visible in panels two and three, thus signifying the typical flux-rope geometry. Note also the comparatively low (about $50000 \mathrm{~K}$ ) temperature in the ICME. The time interval from hours 6 to 7 , and from 8 to 9 shown by the transparent red color bar, are the two periods for which we studied in detail the fluctuations of solar wind velocity and magnetic field and the proton VDFs.

To demonstrate the Alfvénic nature of the fluctuations in the MC, we simply show in Fig. 3 and Fig. 4, respectively, for one hour (corresponding to the two periods marked by the right and left red bars in Fig. 1) the fluctuations of the magnetic-field components and the flow-velocity components. For this goal we applied a rotated coordinate system defined by the hourly averaged magnetic field. For the data of both figures the mean field direction in the old $x-y$-plane defines the new $y^{\prime}$ axis, and thus in the rotated system $x^{\prime}$ is always transverse to the hourly averaged magnetic field, $y^{\prime}$ is directed along it, and finally $z^{\prime}$ is just the same $z$ as in the previous coordinate system. This choice is sufficient for our purpose and case 2, but not ideal for case 1 , where the mean $\mathbf{B}$ still has a finite $z$-component. The fluctuations are 

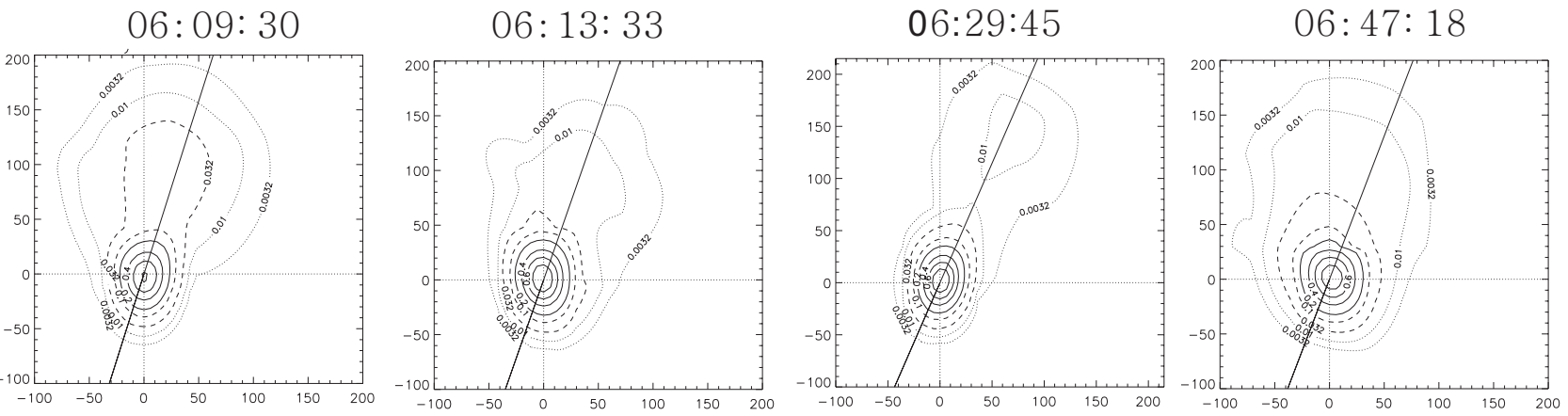

$08: 13: 42$

$08: 24: 30$
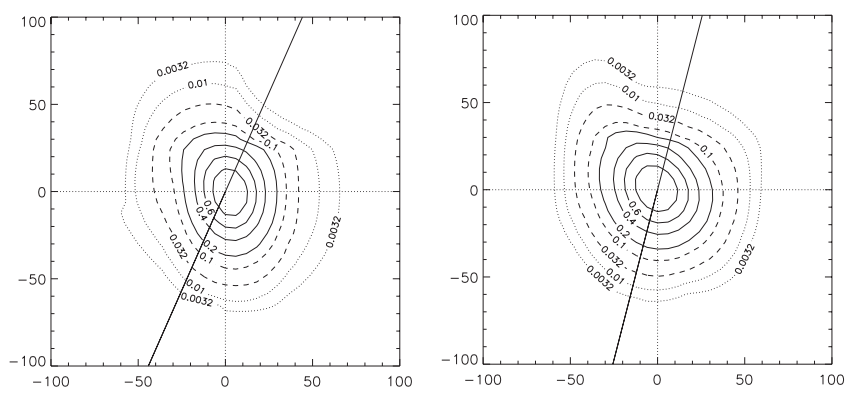

08:39: 21

08: 50: 09
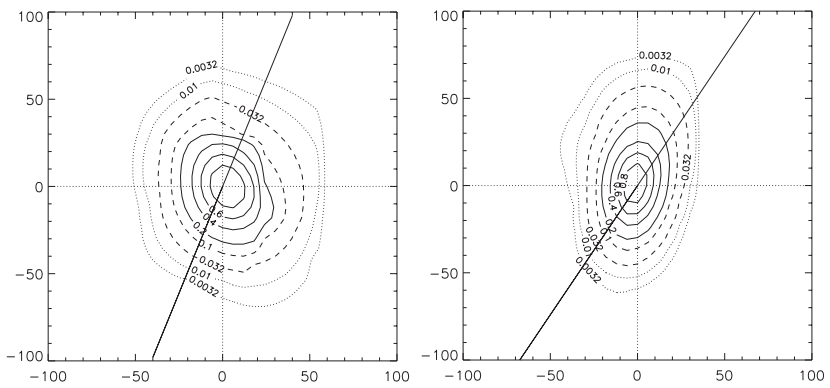

\section{April(DOY 93) 1979}

Fig. 2. Proton velocity distribution functions (VDFs) measured on 3 April 1979 by Helios 2 within an ICME. The ordinate gives the ycomponent and the abscissa the $\mathrm{x}$-component of the proton velocity in $\mathrm{km} / \mathrm{s}$, with the positive $\mathrm{x}$-axis pointing in the radial direction away from the Sun. Note that the frame is set to be rather small to show the distributions more clearly. Actually, their core parts are rather cold. The top row shows four beam or skewed VDFs and the bottom row four cold isotropic VDFs. The typical proton VDF in the top panel has a comparatively cold core part, but in addition an elongated heat flux tail or resolved beam along the local magnetic field direction, which is indicated by the straight line representing the symmetry axis of these gyrotropic VDFs. Note the higher temperature of the beam than the core, clearly visible by much broader isodensity contours, which correspond to 80, 60, 40 and 20 (continuous), 10 and 3.2 (dashed) and 1 and 0.32 (dotted) percent of the maximum phase space density. The relative beam density is only a few percent. The bottom panel shows four cold VDFs, their low temperature being obvious by the narrowly spaced isodensity contours. These distributions are rather typical for the CME proton microstate.

then obtained by taking the differences between the directly measured components and their one-hour average values. All fluctuations are given here in Alfvén velocity units, i.e., we make use of the quantity $\mathbf{V}_{\mathrm{b}}=\mathbf{B} / \sqrt{4 \pi \rho}$, with the magnetic field vector $\mathbf{B}$, and plasma mass density $\rho$. Both quantities are plotted versus time (UT) on 3 April 1979 for the two onehour periods. From top to bottom, the $x^{\prime}, y^{\prime}, z^{\prime}$ vector components and the total magnitudes, respectively, are shown in blue color for the Alfvén velocity and red for the flow velocity in units of $\mathrm{km} / \mathrm{s}$. The fluctuations are considerable, with a typical variance of the corresponding field components at the ten-nT level. In addition, the 1-h mean values of each component are given in Table 1.

We can see in Figs. 3 and 4 that the Alfvénic fluctuations appear most clearly in the $z$ direction, with a typical amplitude of about $50 \mathrm{~km} / \mathrm{s}$. To further analyse these fluctuations in the MC and better identify them as Alfvénic, we made a correlation analysis between the fluctuations of the com- ponents of the solar wind velocity, $\left(\delta V_{s x^{\prime}}, \delta V_{s y^{\prime}}, \delta V_{s z^{\prime}}\right)$ and the Alfvén velocity $\left(\delta V_{b x^{\prime}}, \delta V_{b y^{\prime}}, \delta V_{b z^{\prime}}\right)$. For the time period from 08:00 to 09:00 UT shown in Fig. 4 the number of data points used in this calculation is 43 . The related correlation coefficient for a $95 \%$ confidence level is 0.3 . The calculated correlation coefficients actually are: $c_{x^{\prime}}=0.81$, $c_{y^{\prime}}=0.23$, and $c_{z^{\prime}}=0.81$, respectively, for the three pairs of vector components in the $x^{\prime}, y^{\prime}$ and $z^{\prime}$ direction. And for the time section from 06:00 to 07:00, the number of data points is 24 . The correlation coefficient for a $95 \%$ confidence level is 0.4 . The correlation coefficients are : $c_{x^{\prime}}=0.75, c_{y^{\prime}}=0.39$, and $c_{z^{\prime}}=0.64$. Moreover, the normalized cross helicity values for the two time sections are: 0.55 and 0.64 , respectively. And the values of the normalized residual energy are: -0.73 and -0.63 . We used the normalized cross helicity and residual energy as defined by Bavassano et al. (1998). All these parameters are listed in Table 1. 


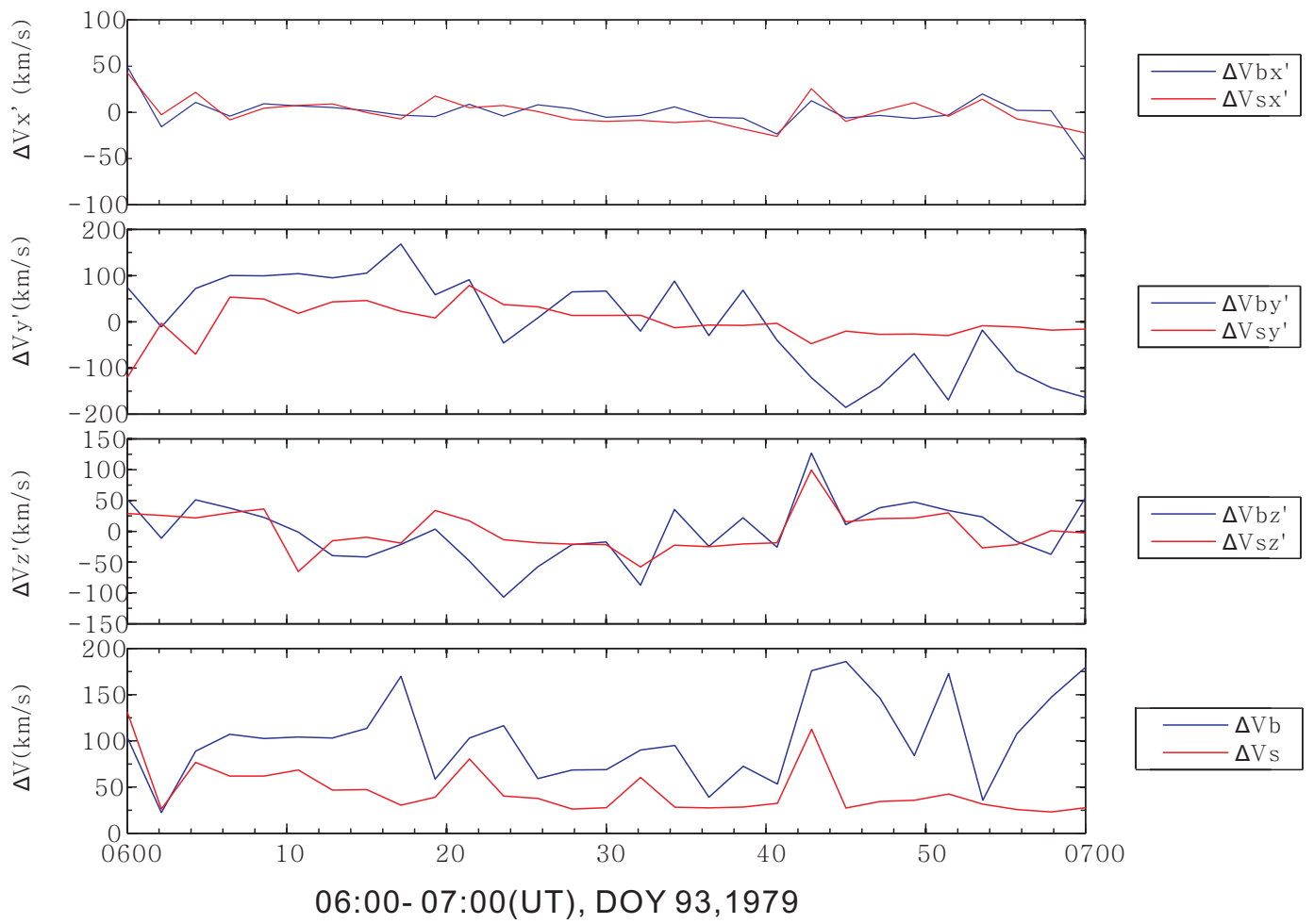

Fig. 3. Fluctuations of the proton velocity and magnetic field vector (displayed as an Alfvén velocity) plotted versus time as measured in the ICME on 3 April between hour 6 and 7 in UT. From top to bottom, the $x^{\prime}, y^{\prime}$ and $z^{\prime}$ components and the total magnitudes are shown (in blue colour the Alfvén and red the flow velocity) in units of $\mathrm{km} / \mathrm{s}$. They indicate sizable overall variations, with temporarily significant transverse correlations indicating Alfvén waves.

We found clear correlations between the velocity and the field components (during the two analysed periods that are shown in Fig. 1). The correlations were fairly high from hour 8 to 9, and somewhat less good from hour 6 to 7 , but yet do reveal the Alfvénic nature of the fluctuations in the ICME. Thus, by inspection of Figs. 3 and 4, one can readily see, particularly in the $x^{\prime}$-component that is transverse to the mean magnetic field, the typical correlations between field and flow which indicate Alfvén waves.

\section{Discussion and conclusions}

We presented here, to our knowledge for the first time, detailed proton VDFs measured inside an ICME. These comparatively cold VDFs are usually characterized by an isotropic core with a very low temperature, but for a 1-h period also by a broad and hot beam propagating along the field direction. The beam lasts for almost an hour and appears to be unusual as compared with the usual ICME proton VDFs that are fairly isotropic. The proton beam corresponds to a thermal anisotropy with larger parallel than perpendicular temperature with respect to the magnetic field. This signature was found before in the driver gas of an ICME reported from ISEE 3 data by Zwickl et al. (1983) and Galvin et al. (1987).

We can presently not say whether proton beams commonly occur in all CMEs, but we plan to do a future statistical investigation of this question. Concerning the general properties of proton beams in the solar wind, especially the dependence of their drift velocity on the proton core plasma beta, see the work by Tu et al. (2004) (also for further relevant references). The nature, origin and characteristics of the proton beam found in the usual slow and fast solar wind in the inner heliosphere (Marsch et al., 1982) and at 1 AU can widely differ, and have been reviewed and discussed in detail by Feldman and Marsch (1997), who argued that proton beams might originate from injections at the base of the expanding corona. Other authors (see the review of Marsch (2006)) suggested that beams in slow streams may have been generated by Coulomb collisions. For fast streams, theoretical arguments were, from instability calculations and numerical simulations, put forward that a proton beam may be caused by Alfvén waves and regulated by electromagnetic instabilities.

The concurrent proton velocity and magnetic field variations in the ICME under study here show the typical signatures of Alfvénic fluctuations. Whereas a wealth of literature 


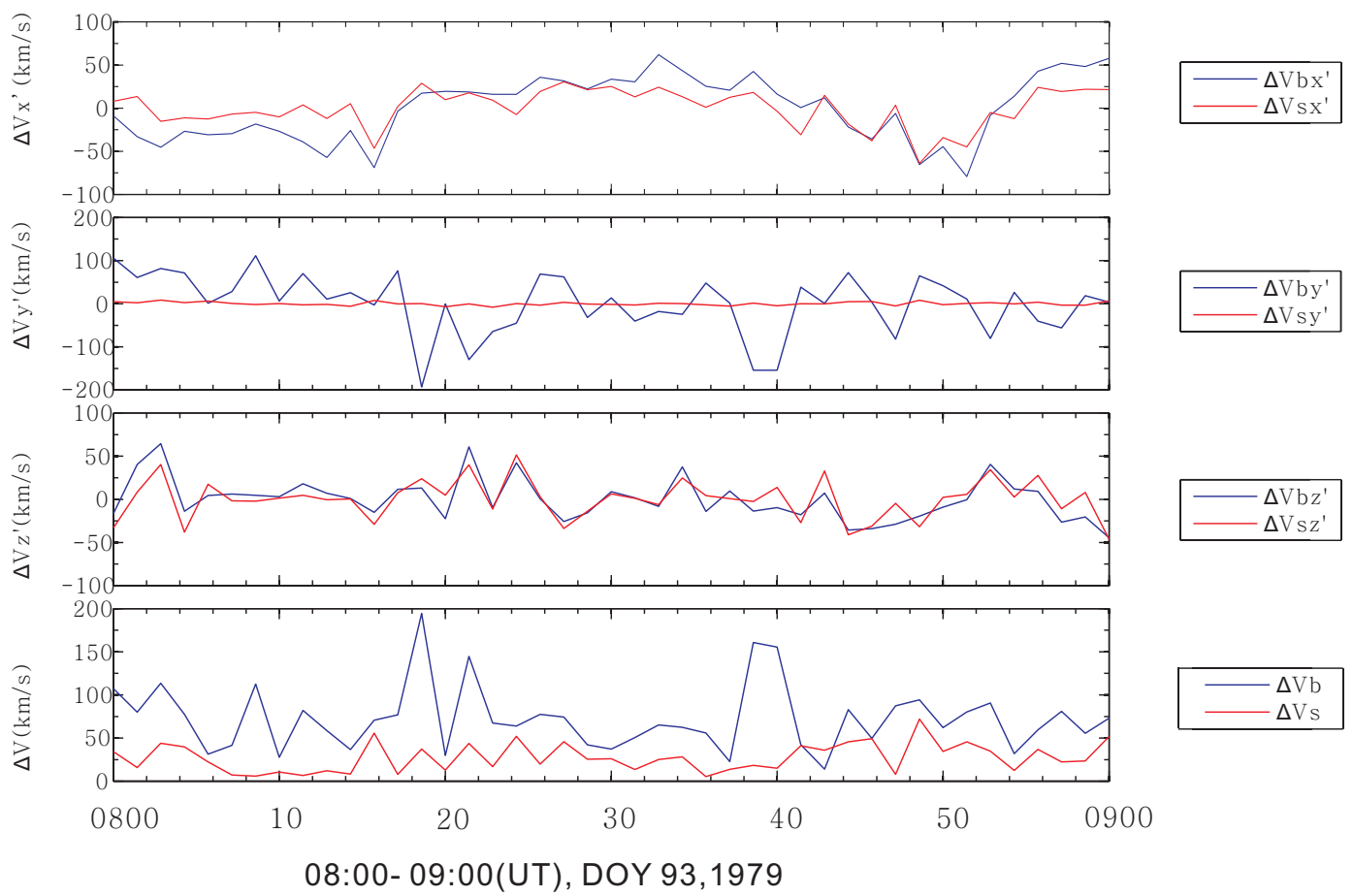

Fig. 4. Fluctuations of the proton velocity and magnetic field vector (displayed as an Alfvén velocity) plotted versus time as measured in the ICME on 3 April between hour 8 and 9 in UT. From top to bottom, the $x^{\prime}, y^{\prime}$ and $z^{\prime}$ components and the total magnitudes are shown (in blue colour the Alfvén and red the flow velocity) in units of $\mathrm{km} / \mathrm{s}$. They indicate sizable overall variations, with good transverse correlations indicating Alfvén waves.

Table 1. Statistical parameters based on hourly averages.

\begin{tabular}{lrr}
\hline Parameter & 06:00-07:00 & 08:00-09:00 \\
\hline Vss $(\mathrm{km} / \mathrm{s})$ & 433.4 & 420.7 \\
Vsy $(\mathrm{km} / \mathrm{s})$ & 203.6 & 147.9 \\
Vsz $(\mathrm{km} / \mathrm{s})$ & -20.1 & -6.3 \\
Vbx $(\mathrm{km} / \mathrm{s})$ & -24.5 & -11.9 \\
Vby $(\mathrm{km} / \mathrm{s})$ & -323.0 & -425.5 \\
Vbz $(\mathrm{km} / \mathrm{s})$ & -167.4 & 1.0 \\
Angle of B with X-axis (degree) & 67.6 & 69.9 \\
Correlation coefficient in $x^{\prime}$ & 0.75 & 0.81 \\
Correlation coefficient in $y^{\prime}$ & 0.39 & 0.23 \\
Correlation coefficient in $z^{\prime}$ & 0.64 & 0.81 \\
Normalized cross helicity & 0.64 & 0.55 \\
Normalized residual energy & -0.63 & -0.73 \\
\hline
\end{tabular}

exist on waves and turbulence in the normal undisturbed solar wind (Tu and Marsch, 1995), comparatively little has been done to investigate small-scale fluctuations in ICMEs. Ruzmaikin et al. (1997) studied the spectral indices of the magnetic field fluctuations in an ICME at scales of minutes as measured on ISEE 3 and found them to be similar to the value of $-5 / 3$ measured in slow streams, but different from the flatter index ranging between -1 and $-5 / 3$ in fast streams.
Leamon et al. (1998) using WIND data determined the geometry of magnetic fluctuations near the local proton gyrofrequency in an ICME and its embedded magnetic cloud, but did not use plasma measurements to show their Alfvénic nature conclusively.

Concerning the beam formation, Tu et al. (2002) have presented a model invoking resonant interaction and pitchangle diffusion of protons with Alfvén/cyclotron waves as the driving force. The beams in the present MC perhaps resulted from such interactions between cold protons and Alfvén waves, which apparently existed in this MC. This formation process is possibly due to a low proton temperature and comparatively low plasma beta. In such plasma conditions, parametric decay processes cause cyclotron and sound waves, which can gradually extract a tail from the core protons and thus lead to a beam (Araneda et al., 2008).

The model proton VDFs resulting from such direct nonlinear numerical simulations resemble the beam ones observed here. However, Alfvénic fluctuations were also found in the second time period from hour 8 to 9 , in which in contrast very isotropic cold VDFs occurred (compare again the periods marked by the two red bars in Fig. 1). Thus, we may question that the proton beam might have been formed locally, and speculate if it is of coronal origin and only stabilized by wave-particle interactions in the ICME on its way 
from the corona to the in situ point of Helios observation. We can of course not eliminate this possibility that the proton beam as such already originated with the CME in the corona.

Acknowledgements. Tu's and Yao's work is supported by the National Natural Science Foundation of China under projects with contract number 40574078, 40436015 and 40336053 and Beijing Education Project with contract number XK100010404 and by the foundation of Major Projects of National Basic Research under contract number G-2006CB806305. S. Yao is supported by the China Scholarship Council for her study in Germany.

Topical Editor R. Forsyth thanks B. Bavassano and A. Szabo for their help in evaluating this paper.

\section{References}

Araneda, J. A., Marsch, E., and Viñas, A. F.: Proton core heating and beam formation via parametrically unstable Alfvén-cyclotron waves, Phys. Rev. Lett., 100(12), 125003, doi:10.1103/PhysRevLett.100.125003, 2008.

Bavassano, B., Pietropaolo, E., and Bruno, R.: Cross-helicity and residual energy in solar wind turbulence: Radial evolution and latitudinal dependence in the region from 1 to $5 \mathrm{AU}$, J. Geophys. Res., 103, 6521-6529, 1998.

Feldman, W. C. and Marsch, E.: Kinetic Phenomena in the Solar Wind, in: Cosmic Winds and the Heliosphere, edited by: Jokipii, J. R., Sonett, C. P., and Giampapa, M. S., Space Science Series, pp. 615-676, Arizona University Press, Tucson, USA, 1997.

Galvin, A. B., Ipavich, F. M., Gloeckler, G., Hovestadt, D., Bame, S. J., Klecker, B., Scholer, M., and Tsurutani, B. T.: Solar wind iron charge states preceding a driver plasma, J. Geophys. Res., 92(A11), 12069-12081, 1987.

Gosling, J. T., Baker, D. N., Bame, S. J., Feldman, W. C., Zwickl, R. D., and Smith, E. J.: Bidirectional solar wind electron heat flux events, J. Geopyhs. Res., 92, 8519-8535, 1987.

Heuer, M. and Marsch, E.: Diffusion plateaus in the velocity distributions of fast solar wind protons, J. Geophys. Res., 112, A03102, doi:10.1029/2006JA011979, 2007.

Kunow, H., Crooker, N. U., Linker, J. A., Schwenn, R., and Von Steiger, R. (Eds.): Coronal Mass Ejections, Space Sciences Series of ISSI, vol. 21, ISBN: 978-0-387-45086-5; Reprinted from Space Sci. Rev., Vol. 123/1-4, 2006.

Leamon, R. J., Smith, C. W., and Ness, N. F.: Characteristics of magnetic fluctuations within coronal mass ejections: The January 1997 event, J. Geophys. Res., 25, 2505, doi:10.1029/98GL00305, 1998.

Marsch, E.: Kinetic Physics of the Solar Corona and Solar Wind, Living Rev. Solar Phys. 3, http://www.livingreviews.org/ lrsp-2006-1, 2006.
Marsch, E., Mühlhauser, K.-H., Schwenn, R., Rosenbauer, H., Pilipp, W., and Neubauer, F.: Solar wind protons: Threedimensional velocity distributions and derived plasma parameters measured between 0.3 and 1 AU, J. Geophys Res., 87, 5272,1982

Marsch, E., Ao, X.-Z., and Tu, C.-Y.: On the temperature anisotropy of the core part of the proton velocity distribution function in the solar wind, J. Geophys. Res., 109, A04102, doi:10.1029/2003JA010330, 2004.

Neubauer, F. M., Beinroth, H. J., Barnsdorf, H., and Dehmel, G.: Initial results from the Helios-1 search-coil magnetometer experiment, J. Geophys., 42, 599-614, 1977.

Rosenbauer, H., Schwenn, R., Marsch, E., Meyer, B., Miggenrieder, H., Montgomery, M. D., Mühlhauser, K.-H., Pillip, W., Voges, W., and Zink, S. M.: A survey of initial results of the Helios plasma experiment, J. Geophys., 42, 561-580, 1977.

Ruzmaikin, A., Feynman, J., and Smith, E. J.: Turbulence in coronal mass ejections, J. Geophys. Res., 102, 19753 , doi:10.1029/97JA01558, 1997.

Skoug, R. M., Feldman, W.C., Gosling, J. T., McComas, D. J., and Smith, C. W.: Solar wind electron characteristics inside and outside coronal mass ejections, J. Geopyhs. Res., 105, 23069, doi:10.1029/2000JA000017, 2000.

Tu, C.-Y. and Marsch, E.: MHD Structures, Waves and Turbulence in the Solar Wind: Observations and Theories, Space Sci. Rev., 73, 1-210, 1995.

Tu, C.-Y., Wang, L.-H., and Marsch, E.: Formation of the proton beam distribution in high-speed solar wind, J. Geophys. Res., 107(A10), 1291, doi:10.1029/2002JA009264, 2002.

Tu, C.-Y., Marsch, E., and Qin, Z.-R.: Dependence of the proton beam drift velocity on the proton core plasma beta in the solar wind, J. Geophys. Res., 109, A05101, doi:10.1029/2004JA010391, 2004.

Wimmer-Schweingruber, R. F.: Coronal Mass Ejections, A Personal Workshop Summary, Space Sci. Rev., 123, 471, doi:10.1007/s11214-006-9025-x, 2006.

Wimmer-Schweingruber, R. F., Crooker, N. U., Balogh, A., Bothmer, V., Forsyth, R. J., Gazis, P., Gosling, J. T., Horbury, T., Kilchenmann, A., Richardson, I. G., Richardson, J. D., Riley, P., Rodriguez, L., von Steiger, R. V., Wurz, P., and Zurbuchen, T. H.: Understanding interplanetary coronal mass ejection signatures, Space Sci. Rev., 123, 177, doi:10.1007/s11214-006-9017$\mathrm{x}, 2006$

Zurbuchen, T. H. and Richardsen, I. G.: In-situ solar wind and magnetic signatures of interplanetary coronal mass ejections, Space Sci. Rev., 123, 31, doi:10.1007/s112214-006-9010-4, 2006.

Zwickl, R. D., Asbridge, J. R., Bame, S. J., Feldman, W. C., Gosling, J. T., and Smith, E. J.: Plasma properties of driver gas following interplanetary shocks observed by ISEE-3, in: Solar Wind Five, NASA Conf. Publ. CP-2280, 711-717, 1983. 